\title{
Coronary artery obstruction associated with congenital aortic valve disease
}

\author{
JOHN L GIBBS, JOHN DAVIES, ^ SHAKEEL A QURESHI† \\ From Harefield Hospital, Harefield, Middlesex
}

SUMMARY Abnormal attachment of the aortic valve caused obstruction to left coronary arterial flow in a young girl, who presented with angina of effort. Aortic valve and root replacement resulted in the patient's effort tolerance returning to normal with resolution of the exercise electrocardiographic and angiographic abnormalities. This case emphasises the importance of an awareness of the existence of such defects and their amenability to surgical correction.

Congenital anomalies of the coronary arteries are uncommon. Abnormalities of the origin, coronary artery fistulas, and hypoplasia of coronary arteries can be diagnosed and are amenable to surgical treatment. Children presenting with angina, syncope, unexplained left ventricular failure, or myocardial infarction require coronary angiography to define the coronary anatomy. We report a case in a young girl, who presented with angina and obstruction of the left main coronary artery due to a congenital aortic valve abnormality producing coronary insufficiency and underwent successful surgery.

\section{Case report}

A 15 year old girl presented with a two month history of angina and dyspnoea of effort and prominent arterial pulsation in the neck. She had been perfectly fit previously, and no murmurs had been reported. She was taking no medication and there was no significant family history.

Physical examination showed a collapsing pulse with a blood pressure of $120 / 60 \mathrm{~mm} \mathrm{Hg}$. There was a thrill over the aortic area radiating to the neck. There was an ejection click followed by a (2/4) systolic ejec-

Requests for reprints to Dr J L Gibbs, Non-Invasive Heart Unit, Killingbeck Hospital, Leeds LS14 6UQ.

^Present address: City General Hospital, Stoke on Trent.

†Present address: Armed Forces Institute of Cardiology, Rawalpindi, Pakistan. tion murmur in the aortic area and a moderately loud and long (4/4) early diastolic murmur of aortic regurgitation. The rest of the physical examination was normal. The chest radiograph showed a normal cardiothoracic ratio and normal lung fields. A resting electrocardiogram showed sinus rhythm, QRS axis of $+30^{\circ}$, and T wave inversion in leads I, II, III, aVF, V5, and V6. The $M$ mode echocardiogram showed a normal appearance of the aortic valve and diastolic flutter of the anterior leaflet of the mitral valve, consistent with aortic regurgitation. The left ventricular fractional shortening was $32 \%$.

She was exercised on the treadmill according to the Bruce protocol. The test was stopped after only three minutes because of angina, and there was almost 2.0 mm ST depression in leads V4-V6 (Fig. 1a). Cardiac catheterisation showed a peak systolic gradient of 20 $\mathrm{mm} \mathrm{Hg}$ across the aortic valve. Aortic root angiography showed grade III/IV aortic regurgitation and an abnormal, tethered membrane like structure arising from one of the aortic valve cusps attached to the free aortic wall just above the origin of the left main coronary artery isolating the left coronary sinus (Fig. 2a). The coronary arteries were entirely normal. There was no evidence of supravalvar aortic stenosis.

At operation under cardiopulmonary bypass the free border of the left coronary cusp was found to be completely fused to the aortic wall above the origin of the left coronary artery with a small opening into the left coronary sinus. The aortic valve could not be repaired, and therefore this and the aortic root were replaced by an aortic homograft by a method 
(a)
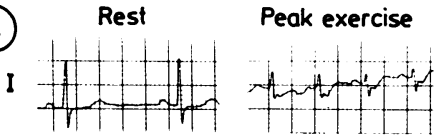

$5 \mathrm{~min}$ after exercise
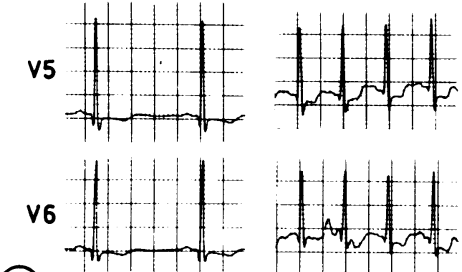

(b)
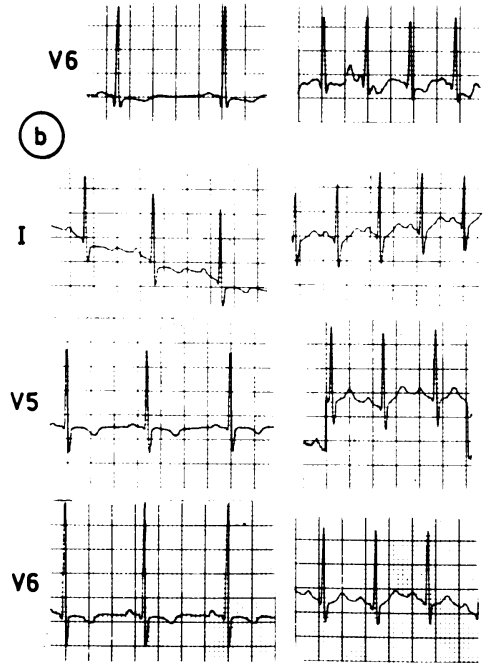

Fig. 1 (a) Preoperative and (b) postoperative electrocardiograms before and after exercise.

described by Thompson $e t$ al. ${ }^{1}$ The coronary arteries were reimplanted.

She made an uneventful recovery from operation, and at review two months later had no symptoms. A resting electrocardiogram showed persistence of the preoperative $T$ wave abnormalities. Treadmill exercise testing was repeated, the patient stopping because of fatigue after 11.5 minutes of the Bruce protocol. The electrocardiogram normalised at peak exercise, the resting $T$ wave abnormalities returning with recovery (Fig. 1b).

Repeat cardiac catheterisation three months postoperatively showed no aortic valve gradient, no evidence of aortic regurgitation (Fig. 2b), and normal coronary arteries.

\section{Discussion}

Angina in children produced by coronary ostial obstruction due to congenital anomalies is rare and may be due to a variety of lesions. Obstruction of the origin of the left coronary artery has been reported in association with supravalvar aortic stenosis as a result of fusion of the left coronary cusp of the aortic valve to
Gibbs, Davies, Qureshi
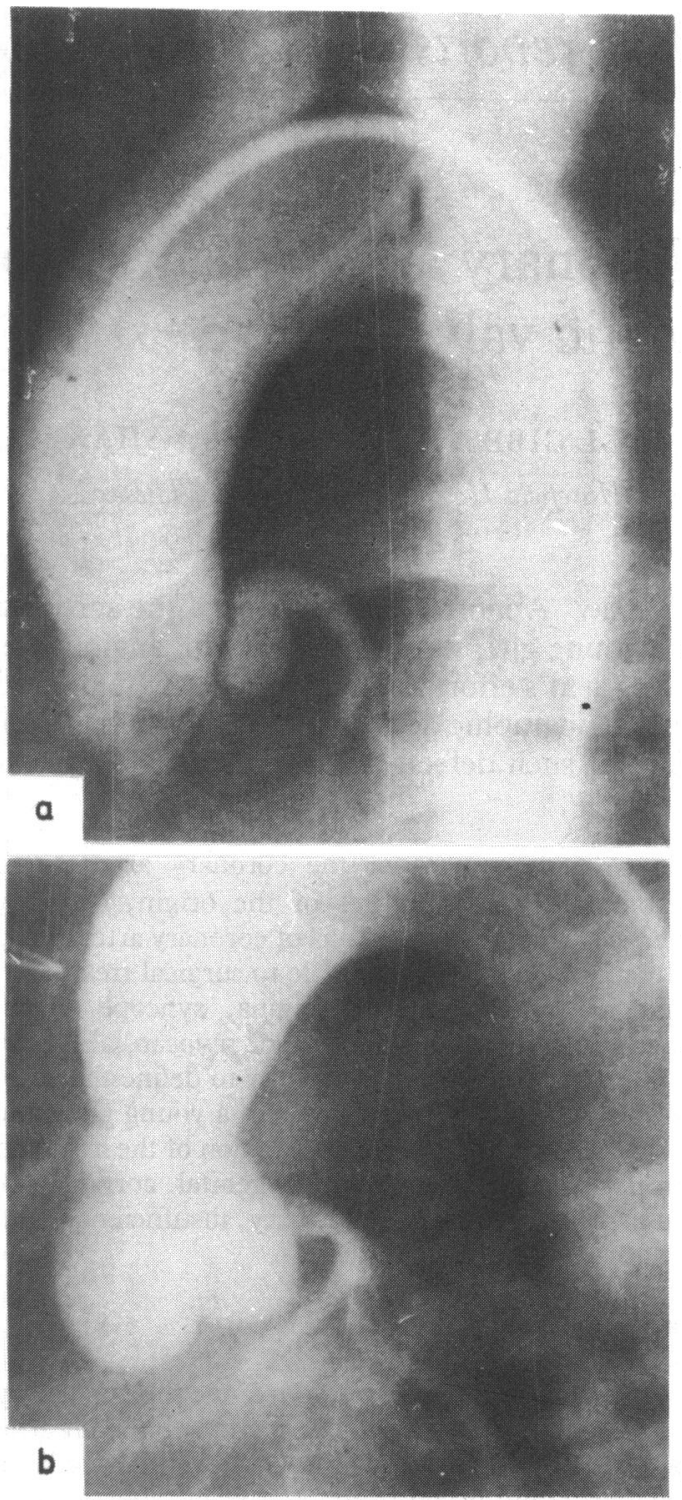

Fig. 2 Aortic root angiograms in the left anterior oblique projection (a) showing the abnormal attachment of the valve cusp and (b) two months after operation.

the area of supravalvar narrowing. ${ }^{2}$ Membraneous obstruction of the left coronary sinus has also been reported in an 8 year old boy in association with congenital discrete subaortic stenosis, ${ }^{3}$ the obstruction being caused by a discrete membrane arising from the aortic wall above the left sinus attached to the left coronary cusp. Line et al reported the case of a 44 year old man with total rather than partial isolation of the 
left coronary sinus by a dysplastic aortic valve cusp, resulting in hypoplasia of the left coronary arterial tree. ${ }^{4}$

There was no evidence of previous infection or inflammation on histological examination of our patient's excised aortic valve, and the cause of the abnormal attachment of the valve leaflet remains obscure.

The presentation of a child or adolescent patient with angina should prompt a search for congenital abnormalities of the coronary arteries or the aortic valve. Unusual causes of coronary arterial obstruction are being reported with increasing frequency, and many of them are suitable for surgical correction. Successful surgery in our patient further emphasises the importance of awareness of the existence of such abnormalities.

We thank Dr Paolo Stassano, Harefield Hospital, for his help in preparing the manuscript, and $\mathrm{Mr}$ Magdi Yacoub and Dr Rosemary Radley-Smith for their valuable advice and permission to report this case.

\section{References}

1 Thompson $\mathrm{R}$, Knight E, Ahmed M, Somerville W, Towers M, Yacoub $M$. The use of "fresh" unstented homograft valves for replacement of the aortic valve. Analysis of 61/2 years' experience. Circulation 1977; 56: 837-41.

2 Kreel I, Reiss R, Strauss L, Blumenthal S, Baronofsky ID. Supra valvular stenosis of the aorta. Ann Surg 1959; 149: 519.

3 Gibson R, Nihill MR, Mullins CE, Cooley DA, Sandiford FM, McNamara DG. Congenital coronary artery obstruction associated with aortic valve anomalies in chiIdren: report of two cases. Circulation 1981; 64: 857-61.

4 Line DE, Babb JD, Pierce WS. Congenital aortic valve anomaly - aortic regurgitation with left coronary artery isolation. $f$ Thorac Cardiovasc Surg 1979; 77: 533-5. 